\title{
ASPECTOS BIO Y CRONOESTRATIGRÁFICOS DEL LEONIENSE (CÁMBRICO MEDIO TEMPRANO) EN LA PENÍNSULA IBÉRICA
}

\author{
Rodolfo GOZALO' y Eladio LIÑÁN \\ 'Departament de Geologia. Universitat de València. Dr. Moliner, 50. E-46100
Burjasot, Valencia. España. Correo electrónico: rodolfo.gozalo@uv.es
2 Departamento de Geología. Universidad de Zaragoza. Ciudad Universitaria.
E-50009 Zaragoza. España.
}

Gozalo, R. y Liñán, E. 1998. Aspectos bio y cronoestratigráficos del Leoniense (Cámbrico Medio temprano) en la Península Ibérica. [Bio and chronostratigraphic aspects of the Leonian (early Middle Cambrian) in the Iberian Peninsula.] Revista Española de Paleontología, nº extr. Homenaje al Prof. Gonzalo Vidal, 97-103. ISSN 02136937.

In Memorian: Esta breve síntesis constituye un pequeño, pero sentido, homenaje al Prof. Gonzalo Vidal Molinero, que nos abandonó prematuramente, y con quien compartimos muchos momentos agradables, tanto de trabajo como de asueto.

\begin{abstract}
The bio and chronostratigraphic data of the Leonian Stage (early Middle Cambrian) from the Iberian Peninsula are analysed in this work to have a more complete characterization of its Paradoxidid zonation. This zonation comprises the following three zones in ascending order: Eoparadoxides mureroensis Zone, Paradoxides (Eccaparadoxides) sdzuyi Zone and Paradoxides (Eccaparadoxides) asturianus Zone. The study of the trilobite assemblages together with the appearance and extinction biohorizons within each zone permit us to present a more accurate correlation within the Leonian strata in Iberian Peninsula.
\end{abstract}

Key words: Trilobites, Leonian (early Middle Cambrian), zonation, Spain.

\section{RESUMEN}

En este trabajo se analizan los datos bio y cronoestratigráficos del Piso Leoniense (Cámbrico Medio temprano) de la Penísula Ibérica con el fin de ofrecer una más completa caracterización de la zonación propuesta en función de las especies de trilobites de la familia Paradoxididae. Esta zonación consta de tres zonas, que por orden de antiguiedad son: Zona de Eoparadoxides mureroensis, Zona de Paradoxides (Eccaparadoxides) sdzuyi y Zona de Paradoxides (Eccaparadoxides) asturianus. El estudio de las asociaciones de trilobites de cada una de estas zonas, así como los biohorizontes de aparición y extinción presentes en ellas, van a permitir una más aquilatada correlación de los estratos leonienses en la Península Ibérica.

Palabras clave: Trilobites, Leoniense (Cámbrico Medio temprano), zonación, España.

\section{INTRODUCCIÓN}

El Cámbrico Inferior y Medio de la Península Ibérica ha sido dividido en distintas unidades bioestratigráficas desde que Lotze (1961) estableciera sus 32 bandas de trilobites con los datos paleontológicos de Sdzuy (1961). Posteriormente Sdzuy (1968) desarrolló una división para el Cámbrico Medio de Asturias, en la que modifica y corrige las bandas anteriores. Poco después, Sdzuy en varios trabajos (197la, b; 1972) establece, para la Península Ibérica, una división formal en pisos para el Cámbrico Inferior e informal para el Cámbrico Medio, en la que perfilaba y aquilataba su propuesta de 1968. Por último Liñán, Perejón y Sdzuy (1993) revisaron los pisos previamente establecidos y definieron los pisos Leoniense y Caesaraugustiense dentro del Cámbrico Medio, completándose así la nomenclatura cronoestratigráfica del Cámbrico Inferior y Medio de la Península Ibérica, actualmente en uso.

Dentro de esta nomenclatura cronoestratigráfica el Piso Leoniense marca, en el sentido clásico, el inicio del Cámbrico Medio en la Península Ibérica; este piso comienza con la aparición en su base de Eoparadoxides mureroensis, y finaliza con la aparición de Badulesia tenera, especie que marca el inicio del Piso Caesaraugustiense (sensu Sdzuy et al., 1996).

Durante esta década los materiales del Piso Leoniense han constituído uno de los objetivos de investigación de 
A- Presa del Porma

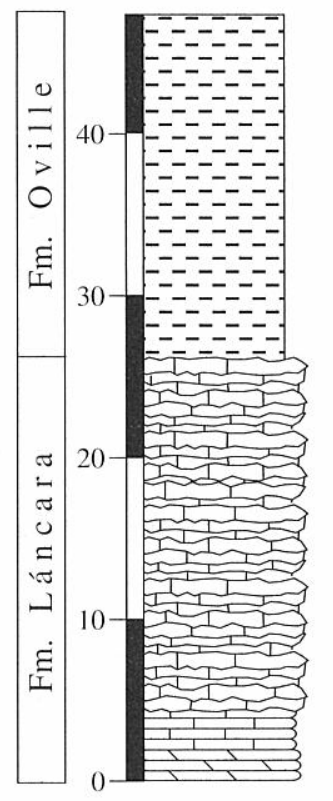

\section{B- Villafeliche 1}

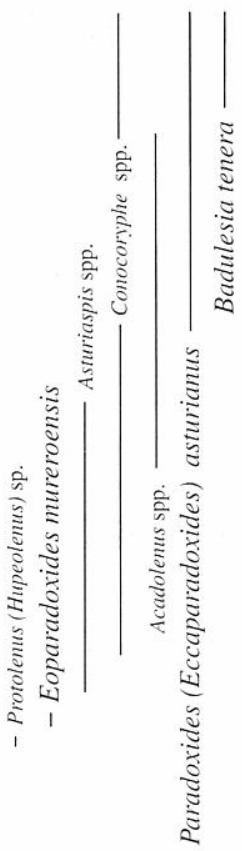

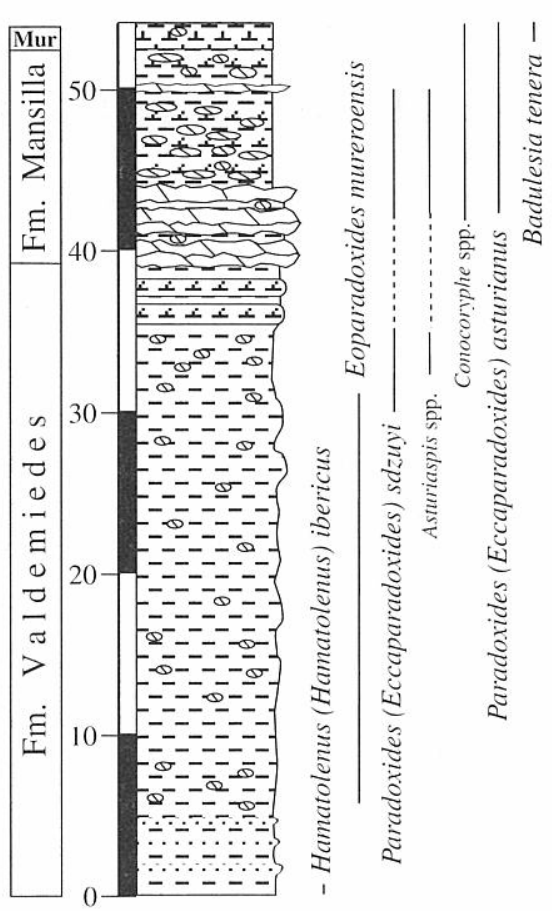

\section{C- Fuente de Bernardo 1}
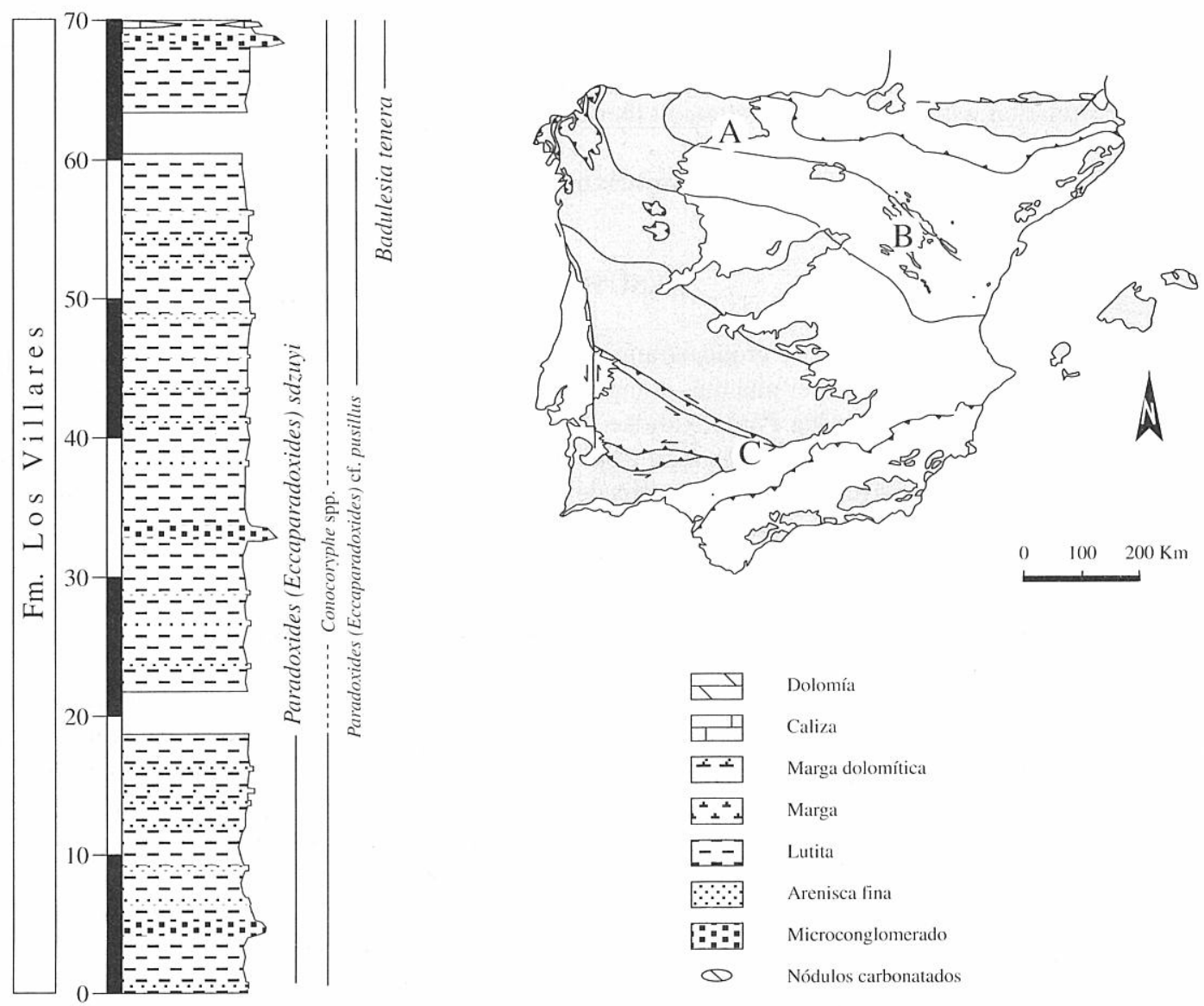

Figura 1. Situación geológica y secciones estudiadas del Leoniense. En las secciones se indica la distribución estratigráfica de las especies índices de las zonas y de los taxones utilizados en la correlación. • A. Sección Presa del Porma (Cordillera Cantábrica, Zona Cantábrica), según Sdzuy (1995). • B. Sección Villafeliche 1 (Cadenas Ibéricas, Zona Asturoccidental-Leonesa), según Gozalo, Liñán y Álvaro (1993). Mur=Formación Murero. • C. Sección Fuente de Bernardo 1 (Sierra de Córdoba, Zona de Ossa-Morena), según Liñán et al. (1995). 
nuestro equipo, para lo cual se han estudiado diversas sucesiones estratigráficas, sus faunas y distribución bioestratigráfica, lo que ha permitido establecer una zonación con paradoxídidos (Gozalo y Liñán, 1995; Liñán et al., 1997) que se va a caracterizar en este trabajo. Además, en este piso se produce la aparición y diversificación de numerosos géneros de trilobites típicos del Cámbrico Medio y, en su parte baja, la desaparición de los últimos representantes de trilobites del Cámbrico Inferior.

Este trabajo pretende ser una puesta al día de la litoestratigrafía y bioestratigrafía de este piso en las diferentes áreas de la Península Ibérica donde afloran materiales de esta edad datados con trilobites (Sierra de Córdoba, Cordillera Cantábrica y Cadenas Ibéricas), y establecer los hitos estratigráficos que permitan realizar las correlaciones entre las diversas áreas.

\section{ESTRATIGRAFÍA}

Los materiales del Leoniense, como ya se ha dicho, se encuentran bien representados en distintas áreas de España (Fig. 1). De cada una de ellas se ha seleccionado la sección, de las ya estudiadas, que presenta la secuencia más completa de los materiales que representan este piso y, además, contiene suficientes trilobites para poder caracterizar adecuadamente las unidades bio y cronoestratigráficas. Las secciones elegidas son Fuente de Bernardo 1 (Sierra de Córdoba, Zona de OssaMorena), Presa del Porma (Cordillera Cantábrica, Zona Cantábrica) y Villafeliche (Cadenas Ibéricas, Zona Asturoccidental-Leonesa).

En estas tres áreas encontramos materiales leonienses en tres facies distintas (Fig. 1). En la Cordillera Cantábrica son facies fundamentalmente carbonatadas (Formación Láncara), aunque en algunas secciones su parte superior se encuentra en facies mixtas y siliciclásticas (base de la Formación Oville). En la Sierra de Córdoba estos materiales están presentes en facies siliciclásticas (Formación Los Villares). Mientras que en las Cadenas Ibéricas lo están en facies mixtas (Grupo Mesones: Formaciones Valdemiedes, Mansilla y Murero).

La posibilidad de estudiar las faunas leonienses en tres facies distintas ha permitido comparar los taxones encontrados en ellas, y seleccionar aquéllos que aparecen en los distintos tipos de facies y que, por lo tanto, presentan una mayor probabilidad de estar registrados y constituyen mejores marcadores bio y cronoestratigráficos que aquéllos que sólo se han encontrado en un tipo de facies.

\section{Sierra de Córdoba}

La única sección, de la Zona de Ossa-Morena, que hasta el momento ha librado trilobites del Leoniense en varios niveles es la sección Fuente de Bernardo 1 (FB1). Esta área ya fue estudiada por Liñán Guijarro (1978), quien estableció la presencia de distintos niveles faunísiticos, aunque debido a la complejidad tectónica no llegó a realizar una columna estratigráfica. Liñán et al.
(1995) revisaron de nuevo esta región y levantaron una columna para estos afloramientos, en los que delimitaron los materiales leonienses de aquellos que pertenecían al Caesaraugustiense. Por último, Perejón et al. (1996) con los datos del trabajo anterior reconocieron en los materiales leonienses las zonas de Paradoxides (Eccaparadosides) sdzuyi y P. (E.) asturianus, además de la de Badulesia tenera, que marca ya el inicio del Caesaragustiense (Fig. 1).

\section{Cordillera Cantábrica}

En estos momentos la única secuencia completa del Leoniense con trilobites publicada de la Zona Cantábrica es la sección de la Presa del Porma (Sdzuy, 1995). En ella, los niveles más altos del Cámbrico Inferior (Zona de Hupeolenus) aparecen cerca del techo de la caliza gris de la Formación Láncara. Dentro de esta misma caliza, en niveles algo superiores, aparece Eoparadoxides mureroensis, que marca ya el inicio del Leoniense. En la suprayacente facies griotte de esta formación, se encuentran varios niveles con trilobites, que están en proceso de estudio. La aparición de Paradoxides (Eccaparadoxides) asturianus Sdzuy, 1968, que marca el inicio de la zona de su mismo nombre, se produce en la base de la Formación Oville (Fig. 1). En niveles superiores de esta Formación aparece Badulesia tenera, que marca el comienzo del Piso Caesaraugustiense.

De todos modos, tanto la Formación Láncara como la base de la Formación Oville presentan un fuerte diacronismo y diferentes secuencias faciales, según los distintos dominios de la Cordillera Cantábrica que han sido reconocidos (Zamarreño, 1972; Sdzuy y Liñán, 1993), en general, el Leoniense está representado en la mayoría de las áreas por la parte superior de la Formación Láncara y, en algunas regiones, por la base de la Formación Oville.

\section{Cadenas Ibéricas}

En la prolongación de la Zona AsturoccidentalLeonesa por las Cadenas Ibéricas hay varias secciones con abundantes trilobites, en las que se han datado materiales del Leoniense, pero la sección más completa y con más niveles faunísticos es la de Villafeliche 1 (Gozalo, Liñán y Álvaro, 1993; Gozalo y Liñán, 1995; Gozalo et al., 1996). En ella, los materiales que representan a este piso son la parte superior de la Formación Valdemiedes y toda la Formación Mansilla, mientras que los niveles basales de la Formación Murero ya pertenecen al Caesaraugustiense (Fig. 1). Además, en esta sección está bien representado el evento Valdemiedes (Liñán, Fernández-Nieto et al., 1993).

\section{BIOESTRATIGRAFÍA}

El Leoniense (anteriormente denominado piso de Acadoparadoxides sensu Sdzuy, 1971b) fue inicialmente dividido en la Cordillera Cantábrica en tres niveles por Sdzuy (1968), los cuales fueron denominados en 1971, de más antiguo a más moderno: "C.i./m." (nivel o niveles 


\begin{tabular}{|c|c|c|c|c|c|}
\hline \multirow{2}{*}{ Series } & \multicolumn{2}{|c|}{ Sdzuy (1971a, b, 1972) } & \multicolumn{2}{|c|}{ Sdzuy et al. (1996) y este trabajo } & \multirow{2}{*}{ BÁLTICA } \\
\hline & Pisos & niveles & Pisos & Zonas & \\
\hline \multirow{5}{*}{ 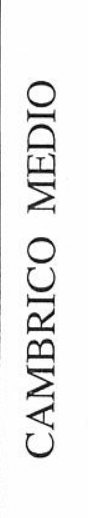 } & \multirow{2}{*}{$\begin{array}{c}\text { Solenople- } \\
\text { uropsidae } \\
\text { (pars) }\end{array}$} & Badulesia tenera & $\begin{array}{l}\text { Caesaraugus- } \\
\text { tiense (pars) }\end{array}$ & Badulesia tenera & $\begin{array}{c}\text { Paradoxides } \\
\text { paradoxissimus (pars) }\end{array}$ \\
\hline & & Badulesia sp. A & \multirow{4}{*}{ Leoniense } & \multirow{2}{*}{$\begin{array}{c}\text { Paradoxides } \\
\text { (Eccaparadoxides) } \\
\text { asturianus }\end{array}$} & \multirow{3}{*}{$\begin{array}{c}\text { Paradoxides } \\
\text { oelandicus }\end{array}$} \\
\hline & \multirow{3}{*}{$\begin{array}{l}\text { Acadopa- } \\
\text { radoxides }\end{array}$} & Acadolenus sp. sp. & & & \\
\hline & & Conocoryphe ovata & & $\begin{array}{c}\text { Paradoxides } \\
\text { (Eccaparadoxides) } \\
\text { sdzuyi }\end{array}$ & \\
\hline & & "C. i. /m." & & $\begin{array}{l}\text { Eoparadoxides } \\
\text { mureroensis }\end{array}$ & \\
\hline \multirow[t]{2}{*}{ C.I. } & \multirow{2}{*}{$\begin{array}{l}\text { Bilbiliense } \\
\quad \text { (pars) }\end{array}$} & & \multirow{2}{*}{$\begin{array}{l}\text { Bilbiliense } \\
\text { (pars) }\end{array}$} & \begin{tabular}{|l|l|}
$\begin{array}{l}\text { Hupe- } \\
\text { olenus }\end{array}$ & $\begin{array}{l}\text { H. }) \text { ibericus } \\
\text { (Honus }\end{array}$ \\
\end{tabular} & \\
\hline & & & & & Proampyx linnarsoni (pars) \\
\hline
\end{tabular}

C.I.: Cámbrico Inferior

"C.i./m.": nivel (o niveles) provisionalmente incluidos en el Cámbrico Medio

Figura 2. Bioestratigrafía del Leoniense de la Península Ibérica y su correlación con la unidades bioestratigráficas de Baltica. (Modificado de Sdzuy et al., 1996.)

asignados con dudas al Cámbrico Medio), Conocoryphe ovata, y Acadolenus sp. sp. Esta división con ligeras variantes se mantuvo en los trabajos posteriores (Fig. 2).

Gozalo y Liñán (1995) proponen una nueva división en tres zonas por medio de las especies de paradoxídidos que se registran en este piso, y que permite un reconocimiento de las mismas en las distintas áreas de la Península Ibérica. Esta zonación (en el sentido de Liñán et al., 1997) ha podido, además, ser contrastada por estos autores en la sección Lemdad 11 del sinclinal de Lemdad en el Anti-Atlas marroquí (Geyer et al., 1995: 96).

En estos momentos el Leoniense comprende tres zonas (Fig. 2), que se desean caracterizar en este trabajo mediante sus asociaciones de trilobites:

\section{Zona de Eoparadoxides mureroensis}

Esta zona fue definida por Liñán, Fernández-Nieto et al. (1993) como Biozona de Paradoxides (Acadoparadoxides) mureroensis; en el mismo sentido fue utilizada por Álvaro et al. (1993), Gozalo, Liñán y Álvaro (1993) y Gozalo, Álvaro et al. (1993). Su límite superior, originalmente establecido con la aparición de Acadolenus, fue modificado por Gozalo y Liñán (1995) para posibilitar el establecimiento de una zonación basada en la filogenia de las especies de paradoxídidos que aparecen en el Leoniense; en este mismo sentido la utilizaron Sdzuy et al. (1996), pero con el nombre de Biozona de Eoparadoxides mureroensis, debido a los cambios de nomenclatura que han afectado al taxón índice, y que también utilizamos en el presente trabajo. Finalmente ha sido definida como zona (Liñán et al., 1997) por su valor cronoestratigráfico.

Está definida como una zona cuyo límite inferior es la aparición del taxón índice, y el límite superior es el nivel anterior al biohorizonte de aparición de Paradoxides (Eccaparadoxides) sdzuyi.

En esta zona, además del taxón índice, se encuentran los siguientes taxones de trilobites: Eoparadoxides sp. indet., Alueva hastata, A. moratrix, A. undulata n. spp., Hamatolenus (Lotzeia) lotzei, Protolenus (Hupeolenus) sp., Olenoides sp., Latoucheia sp., Parasolenopleura sp., Maccanaia sp., Condylopyge cruzensis y Peronopsis spp.

Dentro de ella se produce la extinción de los últimos géneros de trilobites polímeros del Cámbrico Inferior como Hamatolenus, Protolenus, Latoucheia y Alueva, que sobrevivieron a la extinción del límite Cámbrico Inferior/Cámbrico Medio, representada en España por el evento Valdemiedes (Liñán, Fernández-Nieto et al., 1993).

Los bioeventos que caracterizan esta zona son las apariciones de: Eoparadoxides mureroensis, Alueva hastata, A. moratrix y Condylopyge cruzensis, así como las extinciones ya comentadas.

\section{Zona de Paradoxides (Eccaparadoxides) sdzuyi}

Esta zona fue propuesta por Gozalo y Liñán (1995); en el mismo sentido ha sido utilizada por Sdzuy et al. (1996) y en este trabajo.

Está caracterizada como una zona, cuyo límite inferior es la aparición del taxón índice y el límite superior es el nivel previo al biohorizonte de aparición de Paradoxides (Eccaparadoxides) asturianus.

En esta zona se encuentran, además del taxón índice, los siguientes taxones de trilobites: Eoparadoxides mureroensis, Paradoxides (Hydrocephalus) aff. lyelli, P.? enormis, P. (Acaparadoxides) aff. sacheri, Ellipsocephalus bernardi, Corynexochus sp., Olenoides sp., Asturiaspis sp. 1, A. inopinatus, Asturiaspis? sp., Jincella? aff. 
Fuente de Bernardo 1

Villafeliche 1

Presa del Porma
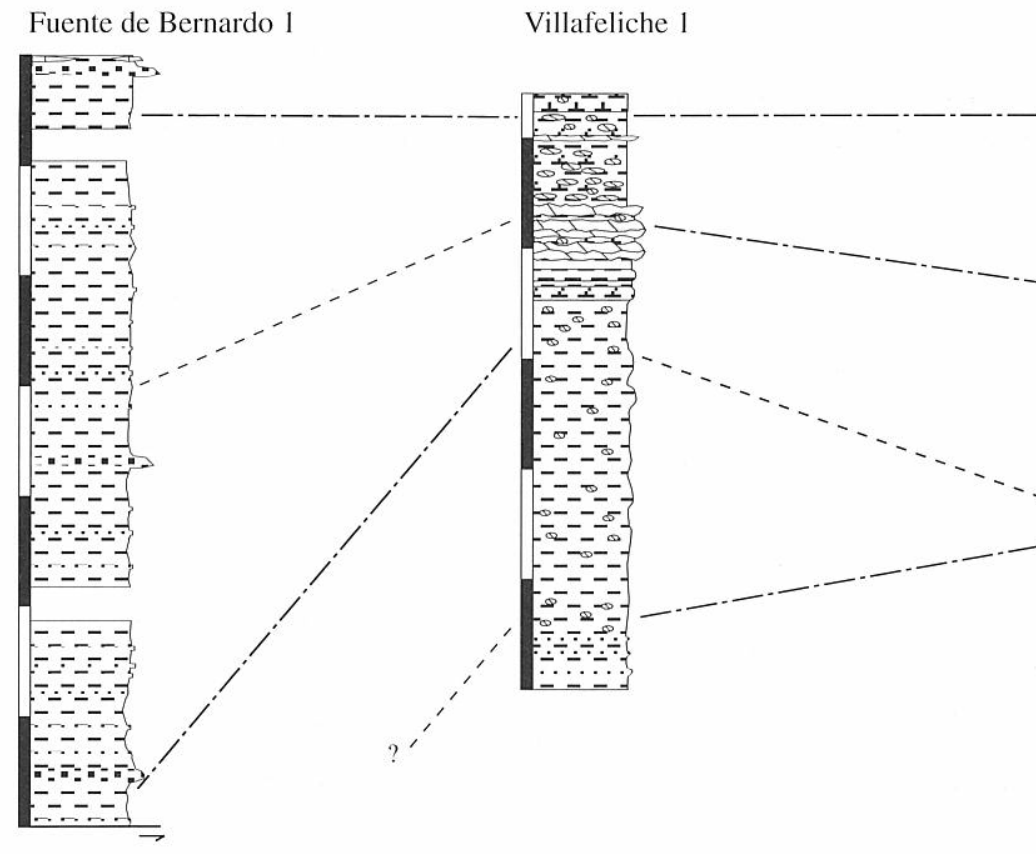

P-----

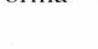

(1)

\begin{tabular}{|c|c|c|}
\hline \multicolumn{2}{|c|}{ Zonas } & Pisos \\
\hline \multicolumn{2}{|c|}{$\begin{array}{c}\text { Badulesia } \\
\text { tenera }\end{array}$} & $\begin{array}{l}\text { CAESARAU- } \\
\text { GUSTIENSE }\end{array}$ \\
\hline \multicolumn{2}{|c|}{$\begin{array}{c}\text { Paradoxides } \\
\text { (Eccapa- } \\
\text { radoxides) } \\
\text { asturianus }\end{array}$} & $\begin{array}{l}\mathrm{L} \\
\mathrm{E}\end{array}$ \\
\hline \multicolumn{2}{|c|}{$\begin{array}{l}\text { Paradoxides } \\
\text { (Eccapa- } \\
\text { radoxides) } \\
\text { sdzuvi }\end{array}$} & $\begin{array}{l}\text { I } \\
\text { E } \\
\text { N } \\
\text { S } \\
\text { E }\end{array}$ \\
\hline \multicolumn{3}{|c|}{ Eoparadoxides } \\
\hline \multicolumn{3}{|c|}{\begin{tabular}{|l|l} 
Huperoelensts & \\
BILBI
\end{tabular}} \\
\hline$\square$ & \multicolumn{2}{|c|}{ Dolomía } \\
\hline प1 & \multicolumn{2}{|c|}{ Caliza } \\
\hline$E$ & \multicolumn{2}{|c|}{ Marga dolomítica } \\
\hline$E+2$ & \multicolumn{2}{|c|}{ Marga } \\
\hline--7 & \multicolumn{2}{|c|}{ Lutita } \\
\hline$\because \because 6$ & Areni & isca fina \\
\hline
\end{tabular}

[:-2 Microconglomerado $\infty$

Nódulos carbonatados

Figura 3. Correlación bioestratigráfica del Leoniense de las secciones Fuente de Bernardo 1, Villafeliche 1 y Presa del Porma.

sulcata, Skreiaspis sp., Acadolenus decorus, A. aff. decorus, Conocoryphe (C.) ovata, C. (Parabailiella) matutina, Bailiella sp. 1, Cornucoryphe schirmi, Condylopyge cruzensis, C. cf. carinata, Peronopsis spp., Dawsonia spp. y Peronopsella prokovskajae prokovskajae.

Los bioeventos que se producen en esta zona y que permiten reconocerla son: la aparición de Paradoxides (Eccaparadoxides) sdzuyi, Paradoxides? enormis, Conocoryphe (C.) ovata, C. (Parabailiella) matutina, Acadolenus decorus, Cornucoryphe schirmi, Asturiaspis inopinatus, Ellipsocephalus bernardi y Peronopsella prokovskajae, y la extinción de Eoparadoxides mureroensis en la parte baja de esta zona.

\section{Zona de Paradoxides (Eccaparadoxides) asturianus}

Esta zona fue propuesta por Gozalo y Liñán (1995) y utilizada, con modificaciones en su límite superior, por Sdzuy et al. (1996); en este último sentido es como se utiliza en este trabajo.

Se caracteriza como una zona, cuyo límite inferior es la aparición del taxón índice y el límite superior es el nivel anterior al biohorizonte de aparición de Badulesia tenera.

En esta zona se encuentran los siguientes taxones de trilobites, además del taxón índice: Paradoxides (Eccaparadoxides) sdzuyi, P. (E.) sulcatus, P. (E.) cf. pusillus, P. (Hydrocephalus) aff. donayrei, P.? enormis, P. (Acaparadoxides) aff. sacheri, Ellipsocephalus bernardi, Corynexochus sp., Jincella? sulcata, Skreiaspis tosali, Acadolenus sp., A. aff. decorus, A. inornatus, Badulesia sp., Dorypyge? sp. Conocoryphe (Parabailiella) matutina, C. (P.) sebarensis, C. (P.) cf. schmidti, C. (P.) languedocensis, Cornucoryphe schirmi,
Bailiella sp., B. cf. tenuicinta, Holocephalina? leve, Bailiaspis sp., B. dalmani, Asturiaspis sp., A. inopinatus, Peronopsis sp., Dawsonia sp., Peronopsella prokovskajae ovetense y $P$. cf. definitus.

Los bioeventos que caracterizan esta zona son: la aparición de Paradoxides (Eccaparadoxides) asturianus, $P$. (E.) sulcatus, Acadolenus inornatus, Jincella? sulcata, Conocoryphe (Parabailiella) sebarensis, C. (P.) languedocensis, Skreiaspis tosali, Holocephalina? leve, Bailiaspis dalmani y Peronopsella prokovskajae ovetense.

\section{CORRELACIÓN}

En la figura 3 se puede observar la correlación de las zonas anteriores en las tres secciones elegidas en este trabajo.

La Zona de Eoparadoxides mureroensis se encuentra representada en las Cadenas Ibéricas y la Cordillera Cantábrica, mientras que en Ossa-Morena no se ha encontrado por el momento. En las dos primeras áreas, a excepción del taxón índice, no se ha encontrado ningún otro trilobites polímero común; sin embargo, en la parte media de la zona aparecen los primeros trilobites miómeros representados por los géneros Condylopyge y Peronopsis, con especies comunes en ambas regiones, lo que unido a la presencia de Maccanaia en la Cordillera Cantábrica posibilita las correlaciones con áreas que no pertenezcan a la Subprovincia Mediterránea (ver Sdzuy, 1995).

La Zona de Paradoxides (Ecccaparadoxides) sdzuyi se encuentra caracterizada por la especie índice en las 
Cadenas Ibéricas y en la Zona de Ossa-Morena, mientras que en la Cordillera Cantábrica todavía no se ha encontrado esta especie, aunque la presencia de especies de Asturiaspis, Conocoryphe y Acadolenus, junto con la presencia de Peronopsella prokovskajae, posibilita establecer una adecuada correlación. Además, la presencia en niveles inferiores y superiores de las especies índices de las zonas infra y suprayacente permiten asegurar con bastante certidumbre esta correlación.

La Zona de Paradoxides (Ecccaparadoxides) asturianus se identifica mediante su especie índice tanto en la Cordillera Cantábrica como en las Cadenas Ibéricas, mientras que en Ossa-Morena, en los niveles equivalentes, encontramos el taxón $P$. (E.) cf. pusillus, especie muy próxima a $P$. (E.) asturianus. Además, como en el caso anterior las especies índices de las zonas infra y suprayacente flanquean los niveles que se equiparan a esta zona.

La Zona de Badulesia tenera, que marca el inicio del Piso Caesaraugustiense, se encuentra bien desarrollada en las tres regiones, y constituye uno de los niveles guías de mayor extensión dentro del Cámbrico Medio, no sólo de la Península Ibérica, sino también de toda la Subprovincia Mediterránea.

\section{AGRADECIMIENTOS}

Este trabajo ha sido financiado por la Dirección General de Estudios Superiores, proyecto PB96-0744. Esta publicación es una contribución al P.I.C.G.: Proyecto 351 Early Paleozoic Evolution in NW Gondwana, y Proyecto 366 Ecological Aspects of the Cambrian Radiation. Agradecemos al Dr. Teodoro Palacios y a José Antonio Gámez (Universidad de Extremadura) sus comentarios al trabajo original.

\section{BIBLIOGRAFÍA}

Álvaro, J., Gozalo, R., Liñán, E. and Sdzuy, K. 1993. The palaeogeography of the northern Iberia at the LowerMiddle Cambrian transition. Bulletin de la Société géologique de France, 164 (6), 843-850.

Geyer, G., Landing, E. and Heldmaier, W. 1995. Faunas and depositional environtments of the Cambrian of the Moroccan Atlas regions. In: Morocco '95. The LowerMiddle Cambrian standard of western Gondwana. Introduction, Field Guide, Abstracts, and Proceedings of the First Conference of the Lower Cambrian Stage Subdivision Working Group and I.G.C.P. Project 366 Ecological Aspects of the Cambrian Radiation. (Eds. G. Geyer and E. Landing). Beringeria, Special Issue 2, 47119.

Gozalo, R. and Liñán, E. 1995. Leonian (early Middle Cambrian) Paradoxides biostratigraphy. In: Morocco '95. The Lower-Middle Cambrian standard of western Gondwana. Introduction, Field Guide, Abstracts, and Proceedings of the First Conference of the Lower Cambrian Stage Subdivision Working Group and
I.G.C.P. Project 366 Ecological Aspects of the Cambrian Radiation. (Eds. G. Geyer and E. Landing). Beringeria, Special Issue 2, 169-171.

Gozalo, R., Álvaro, J., Liñán, E., Sdzuy, K. y Truyols, J. 1993. La distribución de Paradoxides (Acadoparadoxides) mureroensis Sdzuy, 1958 (Cámbrico Medio basal) y sus implicaciones paleobiogeográficas. Cuadernos do Laboratorio Xeolóxico de Laxe, 18, 217 230.

Gozalo, R., Liñán, E. y Álvaro, J. 1993. Bioestratigrafía del Cámbrico Medio de Villafeliche (Prov. Zaragoza, España). Revista Española de Paleontología, $\mathbf{n}^{\circ}$ extraordinario, 49-57.

Gozalo, R., Liñán, E. and Sdzuy, K. 1996. The Lower and Middle Cambrian of Murero and Villafeliche (West Asturian-Leonese Zone. Western Iberian Chain. In: II Field Conference of the Cambrian Stage Subdivision Working Groups. International Subcommission on Cambrian Stratigraphy. Spain, 13-21 September 1996. Field trip guide and abstracts. (Eds. E. Liñán, J. A. Gámez Vintaned and R. Gozalo). Universidad de Zaragoza, Zaragoza, 16-18.

Liñán Guijarro, E. 1978. Bioestratigrafía de la Sierra de Córdoba. Tesis doctorales de la Universidad de Granada, 191, Universidad de Granada, Granada. 212 pp.

Liñán, E., Fernández-Nieto, C., Gámez, J. A., Gozalo, R., Mayoral, E., Moreno-Eiris, E., Palacios, T. y Perejón, A. 1993. Problemática del límite Cámbrico Inferior-Medio en Murero (Cadenas Ibéricas, España). Revista Española de Paleontología, $\mathbf{n}^{\mathbf{0}}$ extraordinario, 26-39.

Liñán, E., Perejón, A. and Sdzuy, K. 1993. The LowerMiddle Cambrian stages and stratotypes from the Iberian Peninsula: a revision. Geological Magazine, 130 (6), 817-833.

Liñán, E., Álvaro, J., Gozalo, R., Gámez-Vintaned, J. A. y Palacios, T. 1995. El Cámbrico Medio de la Sierra de Córdoba (Ossa-Morena, S de España): trilobites y paleoicnología. Implicaciones bioestratigráficas y paleoambientales. Revista Española de Paleontología, 10 (2), 219-238.

Liñán, E., Gonçalves, F., Gámez Vintaned, J. A. y Gozalo, R. 1997. Evolución paleogeográfica del Cámbrico de la Zona de Ossa-Morena basada en el registro fósil. In: Estudo sobre a Geologia da Zona de Ossa-Morena (Maciço Ibérico). Livro de Homenagem ao Professor Francisco Gonçalves. (Eds. A. Alexandre e M. F. Pereira). Universidade de Évora, Évora, 1-26.

Lotze, F. 1961. Das Kambrium Spaniens. Teil I: Stratigraphie. Akademie der Wissenschaften und der Literatur, Abhandlungen der mathematisch-naturwissenschaftlichen Klasse, 1961 (6), 283-498 (1-216). [Traducción por J. Gómez de Llarena: El Cámbrico de España. Memorias del Instituto Geológico y Minero de España, 75, 1-256; año 1970.]

Perejón, A., Moreno-Eiris, E., Liñán, E. and Sequeiros, L. 1996. September 16. The Cambrian of the Sierra de Córdoba. (Ossa-Morena Zone. Sierra Morena). In: II Field Conference of the Cambrian Stage Subdivision Working Groups. International Subcommission on Cambrian Stratigraphy. Spain, 13-21 September 1996. 
Field trip guide and abstracts. (Eds. E. Liñán, J. A. Gámez Vintaned and R. Gozalo). Universidad de Zaragoza, Zaragoza, 45-55.

Sdzuy, K. 1961. Das Kambrium Spaniens. Teil II: Trilobiten. Akademie der Wissenschaften und der Literatur, Abhandlungen der mathematischnaturwissenschaftlichen Klasse, 1961 (7-8), 499-690 (217-408).

Sdzuy, K. 1968. Trilobites del Cámbrico Medio de Asturias. Trabajos de Geología, Universidad de Oviedo, 1 (1967), 77-133.

Sdzuy, K. 1971a. Acerca de la correlación del Cámbrico inferior de la Península Ibérica. I Congreso HispanoLuso-Americano de Geología Económica, Sección 1 Geología, 2, 753-768.

Sdzuy, K. 1971b. La subdivisión bioestratigráfica y la correlación del Cámbrico Medio de España. I Congreso Hispano-Luso-Americano de Geología Económica, Sección 1 Geología, 2, 769-782.

Sdzuy, K. 1972. Das Kambrium der acadobaltischen Faunenprovinz. Zentralblatt für Geologie und Paläontologie, Teil II, 1972 (1-2), 1-91.
Sdzuy, K. 1995. Acerca del conocimiento actual del Sistema Cámbrico y del Límite Cámbrico Inferior-Cámbrico Medio. In: Memorias de las IV Jornadas Aragonesas de Paleontología: "La expansión de la vida en el Cámbrico”. Libro homenaje al Prof. Klaus Sdzuy (Eds. J. A. Gámez Vintaned y E. Liñán). Institución "Fernando el Católico", Zaragoza, 253-263.

Sdzuy, K. y Liñán, E. 1993. Rasgos paleogeográficos del Cámbrico Inferior y Medio del norte de España. Cuadernos do Laboratorio Xeolóxico de Laxe, 18, 189215.

Sdzuy, K., Liñán, E. and Gozalo, R. 1996. The Middle Cambrian Series. In: II Field Conference of the Cambrian Stage Subdivision Working Groups. International Subcommission on Cambrian Stratigraphy. Spain, 13-21 September 1996. Field Trip Guide and Abstracts. (Eds. E. Liñán, J. A. Gámez Vintaned and R. Gozalo). Universidad de Zaragoza, Zaragoza, 16-18.

Zamarreño, I. 1972. Las litofacies carbonatadas del Cámbrico de la Zona Cantábrica (NW de España) y su distribución paleogeográfica. Trabajos de Geología, Universidad de Oviedo, 5, 1-118.

Manuscrito recibido: 31 de julio, 1997 Manuscrito aceptado: 15 de junio, 1998 